coronary arteries were assessed by Gensini scoring system. The fasting serum concentrations of CatS and CysC were measured before angiography respectively. The SPSS11.0 system was applied to analyse the data. Compared with controls, serum levels of cathepsin $\mathrm{S}$ in $\mathrm{CAD}$ groups increased significantly and demonstrated positive correlation $(\mathrm{r}=0.69, \mathrm{p}=0.0001)$ with Gensini score. After being corrected for other common factors (age, sex, blood pressure and blood lipid, etc) by multivariate stepwise regression analysis, this relationship also existed.

Conclusions Serum levels of cathepsin S are associated with severity of athero sclerostic lesions of coronary arteries. The higher serum levels of cathepsin S are, the severer atherosclerostic lesions of coronary arteries are

\section{e0396 CLINICAL ANALYSIS OF 30 INDIVIDUALS WITH MICRO CK TYPE 1}

doi:10.1136/hrt.2010.208967.396

Zhi-Yuan Dong, Zhi-kai Wang, Ji-Yong Liu, Xiao-Bin Tang, Li-Mei Wang, Lian-Yi Shi, Yuan-Ying Liu, Xiu-Zhen Zhao. Healthcare Center, The Central Hospital of CNPC, Langfang

Objective The aim of the prospective study was to investigate the clinical and laboratory features of individuals with micro creatine kinase type 1 (MCK-1), and to evaluate the clinical significance of these cases who expressed serum MCK-1.

Methods To screen MCK-1individuals from serum samples in our biochemistry laboratory from April 2002 to April 2010 according to both increasing CK-MB activity and CK-MB/CKratio over $25 \%$, and finally confirmed by agarose gel electrophoresis of CK isoenzyme analysis. To analyse the clinical and laboratory data of individuals who expressed serum MCK-1 isoenzyme.

Results MCK- 1 was detected in the samples from 30 subjects. Their mean age at the initial presentation was 58.6 years (range 26 to 81), $63.3 \%$ were female $(n=19)$, and $39.3 \%(n=11)$ had hypertension. of the 30 subjects, 10 (33.3\%) had increased total CK activity, and $7(23.3 \%)$ showed the abnormal phenomenon that CK-MB:CK ratio was $>1$. Our study indicated that the misdiagnostic rate was $40.0 \%$ $(n=12)$, and the rate of missed diagnosis was $46.7 \%(n=14)$. There were 3 deaths during the follow-up period.

Conclusion MCK-1 was not rare in clinical practice. Among the conditions in which CK-MB activity is elevated in the absence of myocardial injury or infarction using immunoinhibition methods, MCK-1 merits special attention from clinicians. CK-MB index is a simple and rapid screening test for MCK-I.

\section{e0397 A CORRELATIONAL STUDY OF ACE GENE POLYMORPHISMS}

doi:10.1136/hrt.2010.208967.397

${ }^{1}$ Yun Meiling, ${ }^{2}$ Zheng Yin, ${ }^{3}$ Zhong Jianghua, ${ }^{3}$ Zeng Yu, ${ }^{3}$ Li Tianfa, ${ }^{4}$ Zhang Yong. ${ }^{1}$ Affiliated Hospital; Hainan Medical College; ${ }^{2}$ Hainan Provincial Peoples Hospital; ${ }^{3}$ Affiliated Hospital Hainan Medical College; ${ }^{4}$ Hainan Province Diseases of The Aged Hospital

Objective To study the correlation of the ACE gene polymorphisms with coronary heart disease (CHD) in the Hainan Li nationality.

Methods Used the PCR to detect the polymorphisms of ACE gene insertion/deletion (I/D) in 150 patients with CHD and 150 healthy people from Hainan Li nationality. The genotype frequencies and allele frequencies of DD, DI and II were observed. Specific PCR detection was performed for patients who have been determined as DD by normal PCR to reduce misclassification rate.

Results In the CHD group of Hainan Li nationality, the genotype frequencies of DD, DI and II were $24.7 \%, 32.7 \%, 42.6 \%$ respectively, and the allele frequencies of $\mathrm{D}$ and I were $41.0 \%$ and $59.0 \%$. In the healthy control of Hainan Li nationality, the genotype frequencies of DD, DI and II were $14.0 \%, 44.0 \%, 42.0 \%$ respectively, and the allele frequencies of $\mathrm{D}$ and I were $36.0 \%$ and $64.0 \%$. There were significant differences both in the genotype frequencies of DD, DI and II, and in allele frequencies $D$ and I between these two groups $(p<0.05)$. There were no significant differences in the age, the blood pressure, the body mass index (BMI), the total serum cholesterol (TC) and the low density lipoprotein cholesterol (LDL-C) between these two groups $(p>0.05)$. There was significant differences in the sex between these two groups $(p<0.05)$. The triglyceride (TG) level in the CHD group was significantly higher than in the control $(p<0.05)$. The high density lipoprotein cholesterol (HDL-C) level in the CHD group was significantly lower than the control $(p<0.05)$. Conclusions There were notable correlation between the ACE gene $\mathrm{I} / \mathrm{D}$ polymorphisms and the CHD in Hainan Li nationality. The ACE gene polymorphisms are the major gene that causes CHD in Hainan Li nationality. It may be the independent risk factor in CHD in Hainan Li nationality. So it is very important to intervene with ACEI inhibitors or angiotensin receptor blockers (ARB) in the early stage of $\mathrm{CHD}$.

\section{e0398 A STUDY THE RELATION OF ACE GENE POLYMORPHISMS AND RISK FACTOR WITH CORONARY HEART DISEASE IN HAINAN LI AND HAN NATIONALITY}

doi:10.1136/hrt.2010.208967.398

${ }^{1}$ Yun Eilingm, ${ }^{2}$ Zheng Yin, ${ }^{3}$ Zhong Janghua, ${ }^{3}$ Zeng Yu, ${ }^{3}$ Li Tianfa, ${ }^{4}$ Wang Zhen. ${ }^{1}$ Affiliated Hospital; Hainan Medical College; ${ }^{2}$ Hainan Provincial Peoples Hospital; ${ }^{3}$ Affiliated Hospital Hainan Medical College; ${ }^{4}$ Hainan Province Diseases of The Aged Hospital

Objective To probe into the relation of the ACE gene polymorphisms and the risk factor with coronary heart disease (CHD) in the Hainan $\mathrm{Li}$ and Han nationality.

Methods Used the PCR to detect the polymorphisms of ACE gene insertion/deletion (I/D) in 150 patients with CHD and 150 healthy people from Hainan $\mathrm{Li}$ and Han nationality. The genotype frequencies and allele frequencies of DD, DI and II were observed. Specific PCR detection was performed for patients who have been determined as DD by normal PCR to reduce misclassification rate. Results In the CHD group of Hainan Li nationality, the genotype frequencies of DD, DI and II were $24.7 \%, 32.7 \%, 42.6 \%$ respectively, and the allele frequencies of $\mathrm{D}$ and I were $41.0 \%$ and $59.0 \%$. In the healthy control of Hainan Li nationality, the genotype frequencies of DD, DI and II were $14.0 \%, 44.0 \%, 42.0 \%$ respectively, and the allele frequencies of $\mathrm{D}$ and I were $36.0 \%$ and $64.0 \%$. There were significant differences both in the genotype frequencies of DD, DI and II, and in allele frequencies $D$ and I between these two groups $(p<0.05)$. In the CHD group of Hainan Han nationality, the genotype frequencies of $\mathrm{DD}, \mathrm{DI}$ and II were $23.3 \%, 45.4 \%, 31.3 \%$ respectively, and the allele frequencies of $\mathrm{D}$ and I were $46.0 \%, 54.0 \%$. In the healthy control of Hainan Han nationality, the genotype frequencies of DD, DI and II were $13.3 \%, 42.0 \%, 44.7 \%$ respectively, and the allele frequencies of $\mathrm{D}$ and I were $34.3 \%, 65.7 \%$. There were significant differences both in the genotype frequencies of DD, DI and II, and in allele frequencies D and I between these two groups $(p<0.05)$. The multiple logistic regression analysis showed that the triglyceride (TG) level in the CHD group was significantly higher than in the control $(p<0.05)$ and the high density lipoprotein cholesterol (HDLC) level in the CHD group was significantly lower than the control in both nationality $(p<0.05)$.

Conclusions There were notable correlation between the ACE gene $\mathrm{I} / \mathrm{D}$ polymorphisms and the CHD in Hainan Li and Han nationality. The ACE gene polymorphisms are the major gene that causes the 
CHD in Hainan Li and Han nationality. The higher TG level and the lower HDL-C level may be the risk factor in Hainan Li and Han nationality.

\section{E0399 THE ACE GENE POLYMORPHISMS DISTRIBUTION STATUS IN HAINAN LI AND HAN PEOPLES WITH CORONARY HEART DISEASE}

\author{
doi:10.1136/hrt.2010.208967.399
}

${ }^{1}$ Yun Meiling, ${ }^{2}$ Zhong Janghua, ${ }^{3}$ Zheng Yin, ${ }^{2}$ Zeng Yu, ${ }^{2}$ Li Tianfa, ${ }^{4}$ Zhang Yong. ${ }^{1}$ Affiliated Hospital; Hainan Medical College; ${ }^{2}$ Affiliated Hospital Hainan Medical College; ${ }^{3}$ Hainan Provincial Peoples Hospital; ${ }^{4}$ Hainan Province Diseases of The Aged Hospital

Objective To explore the significance of the ACE gene insertion/ deletion (I/D) polymorphism in peoples of Hainan $\mathrm{Li}$ and $\mathrm{Han}$ nationality with coronary heart disease (CHD).

Methods Used the PCR to detect the polymorphisms of ACE gene insertion/deletion (I/D) in 150 patients with CHD and 150 healthy people from Hainan Han and Li nationality respectively. Observed the genotype frequencies and allele frequencies of DD, DI and II. Specific PCR detection was performed for patients who have been determined as DD by normal PCR to reduce misclassification rate. Meanwhile detected the blood lipid, the lipoprotein, the blood pressure, the blood sugar in all people. Used the multiple regression analysis to find out the risk factor in CHD patients.

Results The genotype frequencies of DD in the CHD group of Han and Li nationality are significantly higher than the control group of $\mathrm{Han}$ and $\mathrm{Li}(\mathrm{p}<0.05)$. There were no significant differences in the genotype frequencies of DD, DI and II between the Han and Li with CHD. By the multiple regression analysis it shows: the genotype frequencies of DD in the CHD group of Han and Li nationality increased, the high density lipoprotein cholesterol (HDL-C) level in the CHD group of Han and Li nationality decreased. The triglyceride (TG) level in the CHD group of Han nationality increased.

Conclusions The genotype frequencies of DD are associated with CHD. The susceptibility of CHD in Han and Li nationality is the same. Increasing the HDL-C level can protect the $\mathrm{CHD}$ patients. The high level of TG is the independent risk factor in Hainan Han people with CHD.

\section{e0400 MOBILISE AUTOLOGOUS BONE MARROW STEM CELLS TO REPAIR INFARCTED MYOCARDIUM}

doi:10.1136/hrt.2010.208967.400

Bo Wang, Xiaojuan Bai. The First Affiliated hospital of China Medical University

Objective Autologous bone marrow stem cells were mobilised and released by cytokines. On the basis of homing and injured microenvironment theories, we investigated the effective reparation of situ transplantation for acute myocardial infarction in rats.

Methods (1) We divided 60 Wistar rats into situ transplantation groug and control group; To duplicate rat' acute myocardial infarction model by injection of drug; CK, LDH level were checked by automatic biochemistry analyser; (2) 30 Wistar rats were injected rhG-CSF $50 \mu \mathrm{g} / \mathrm{kg}$.day; (3) We did a control analysis on pathological section between two groups by histological staining technique and computer graphic analysis; (4) Heart function were checked by polygraph system after AMI model 4 weeks.

Results (1) Serum cardiac enzymes were higher after AMI model $48 \mathrm{~h}$ than it before AMI model. We saw local necrosis region with grid and trabs shape in endocardium of left ventricular apex and papillary muscle; (2) The infarction size in situ transplantation group was smaller than one in control group; (3) The heart function parameters improved significantly in situ transplantation group compared with control group.

Conclusions (1) Rat's acute myocardial infarction model could be completed by injecting $10 \mathrm{mg} / \mathrm{kg}$ isoprenaline interaperitoneally; (2) Infarcted myocardium were repaired and heart function improved by using cytokines.

\section{C0401 CHANGES OF INTRACELLULAR CALCIUM CONCENTRATION IN CARDIAC-LIKE MYOCYTES}

doi:10.1136/hrt.2010.208967.401

Wang bo, Bai xiaojuan. The First Affiliated hospital of China Medical University

Objective To study the effects of verapamil, endothelin on $\left[\mathrm{Ca}^{2+}\right]_{\mathrm{i}}$ in cardiac-like myocytes derived of bone marrow mesenchymal stem cells.

Methods (1) Bone marrow mesenchymal stem cells and cardiac myocytes were cultured by primary method; (2) Bone marrow mesenchymal stem cells differentiated into cardiac-like myocytes by 5-azacytidine induction agent; (3) We divide our test into three groups, including the first generation bone marrow mesenchymal stem cells, cardiac-like myocytes and cardiac myocytes; (4) The alteration of $\left[\mathrm{Ca}^{2+}\right]_{\mathrm{i}}$ affected by verapamil, endothelins was observed with $\left[\mathrm{Ca}^{2+}\right]_{i}$ fluorescence imagine system in bone marrow mesenchymal stem cells, cardiac-like myocytes and cardiac myocytes.

Results (1) Cardiac myocytes were set up through trypsogen digestion method; (2) The alteration of $\left[\mathrm{Ca}^{2+}\right]_{\mathrm{i}}$ affected by verapamil was observed with $\left[\mathrm{Ca}^{2+}\right]_{\text {i }}$ fluorescence imagine system. There was a same trend of variability between cardiac-like myocytes and cardiac myocytes, but no changes in bone marrow mesenchymal stem cells; (3) The alteration of $\left[\mathrm{Ca}^{2+}\right]_{\mathrm{i}}$ affected by endothelin was observed with $\left[\mathrm{Ca}^{2+}\right]_{\mathrm{i}}$ fluorescence imagine system. There was a same trend of variability that fluorescence intensity gradually strengthened with intervention time extended.

Conclusions After affected by verapamil and endothelins, there was a same trend of $\left[\mathrm{Ca}^{2+}\right]_{\mathrm{i}}$ changes between cardiac-like myocytes from bone marrow mesenchymal stem cells and cardiac myocytes. It shows that some common electrophysiological characteristics exist in cardiac-like myocytes and cardiaomyocytes.

\section{e0402 THE EXPRESSION AND RELATION OF THE VITAMIN D3 UP. REGULATED PROTEIN 1 IN PERIPHERAL BLOOD MONONUCLEAR CELLS FROM PATIENTS WITH CORONARY ARTERY DISEASE}

doi:10.1136/hrt.2010.208967.402

Shui Xing, Wu Zhen. The Third Affiliated Hospital of Sun Yat-sen University

Aims Vitamin D3 up-regulated protein 1(VDUP1) is a stressresponse gene and participates in oxidative stress, inflammation, apoptosis, proliferation, glucose homeostasis and lipid metabolism. All of these biological effects play important roles in atherosclerosis. Hence, we made an attempt to study the gene expression of VDUP1 using PBMCs from patients with coronary artery disease (CAD).

Methods The total RNA of PBMCs were acquired from 20 normal persons without history of cardiovascular disease and 72 patients with $\mathrm{CAD}$. The $\mathrm{CAD}$ group was divided into 6 subgroups judged by following risk factor. The subgroup 1 was patient without hyperlipidaemia, hypertension and diabetes. The subgroup 2 was patient with hypertension only. The subgroup 3 was patient with hyperlipidaemia only. The subgroup 4 was patient with diabetes only. The subgroup 5 was patient with hyperlipidaemia plus hypertension. 